(C) [2009] IEEE. Reprinted, with permission, from [Jordan S. Nguyen, Thanh H. Nguyen, Hung T. Nguyen, Semiautonomous Wheelchair System Using Stereoscopic Cameras, Engineering in Medicine and Biology Society, 2009. EMBC 2009. Annual International Conference of the IEEE, 3-6 Sept. 2009]. This material is posted here with permission of the IEEE. Such ermission of the IEEE does not in any way imply IEEE endorsement of any of the University of Technology, Sydney's products or services. Internal or personal use of this material is permitted. However, permission to reprint/republish this material for advertising or promotional purposes or for creating new collective works for resale or redistribution must be obtained from the IEEE by writing to pubs-permissions@ieee.org. By choosing to view this document, you agree to all provisions of the copyright laws protecting it 


\title{
Semi-autonomous Wheelchair System Using Stereoscopic Cameras
}

\author{
Jordan S. Nguyen, Thanh H. Nguyen, Hung T. Nguyen, Senior Member, IEEE
}

\begin{abstract}
This paper is concerned with the design and development of a semi-autonomous wheelchair system using stereoscopic cameras to assist hands-free control technologies for severely disabled people. The stereoscopic cameras capture an image from both the left and right cameras, which are then processed with a Sum of Absolute Differences (SAD) correlation algorithm to establish correspondence between image features in the different views of the scene. This is used to produce a stereo disparity image containing information about the depth of objects away from the camera in the image. A geometric projection algorithm is then used to generate a 3Dimensional (3D) point map, placing pixels of the disparity image in 3D space. This is then converted to a 2-Dimensional (2D) depth map allowing objects in the scene to be viewed and a safe travel path for the wheelchair to be planned and followed based on the user's commands. This assistive technology utilising stereoscopic cameras has the purpose of automated obstacle detection, path planning and following, and collision avoidance during navigation. Experimental results obtained in an indoor environment displayed the effectiveness of this assistive technology.
\end{abstract}

\section{INTRODUCTION}

$\mathrm{P}$ OWER wheelchairs are necessary for assisting in the mobility of severely disabled people. However this assistance is not sufficient enough in many cases of severe disability to provide mobility that does not depend on a caregiver. To overcome this issue certain wheelchairs have been fitted with equipment used in robotics, such as sensors, and given new, generally autonomous, capabilities such that they are put in the category of 'smart wheelchairs'. These have varying uses and assistive capabilities in order to enhance the independence and mobility of severely disabled people [1-2].

As independence comes with being in control, users will tend to prefer systems as close to the manual side of the scale as possible. However, the degree of the user's disability is what defines how far along the autonomous capabilities scale is required. Focused on in this project is the semi-autonomous aspect where environments are unknown to the navigation system. Some 'smart wheelchair' systems that have been commercialised to-date include the

This work was supported in part by Australian Research Council under Discovery Grant DP0666942 and LIEF Grant LE0668541.

Jordan S. Nguyen is with Faculty of Engineering and Information Technology, University of Technology, Sydney, Broadway, NSW 2007, Australia (phone: +612-9514-2451; fax: +61 29514 2868; e-mail: Jordan.Nguyen@uts.edu.au).

Thanh H. Nguyen is with Faculty of Engineering and Information Technology, University of Technology, Sydney, Broadway, NSW 2007, Australia (e-mail: thnguyen@eng.uts.edu.au).

Hung T. Nguyen is with Faculty of Engineering and Information Technology, University of Technology, Sydney, Broadway, NSW 2007, Australia (e-mail: Hung.Nguyen@uts.edu.au).
NavChair Assistive Wheelchair Navigation System which utilises ultrasonic sensors [3], the Hephaestus Smart Wheelchair System which utilises sonar sensors [4], and the Bremen Autonomous Wheelchair also using ultrasonic sensors [5].

More recent autonomous and semi-autonomous wheelchair developments tend to use laser range finders, however these can be very expensive. The most common laser sensors used provide fast and accurate distances to objects, but only on a 2D plane [1]. They are often used in conjunction with other sensors or for localisation in known environments. The wheelchair development of this project aims to produce a wheelchair which can navigate semiautonomously using stereoscopic cameras, which is more suitable for the target market of severely disabled people. Today vision is becoming a more attractive choice since cameras are becoming cheaper and are being used in more applications. They are compact, accurate, and can be used to provide useful information about an environment. They are also a sensor people can relate to easily given that vision is the sense primarily used by humans and animals to perceive their environment and navigate [6].

This paper describes the development of a semiautonomous wheelchair system using stereoscopic cameras to assist hands-free control technologies such as head movement controllers and brain-computer interfaces. Section II provides an overview of the methods used in the design and developments, mainly those associated with the use of the Point Grey Research Bumblebee XB3 stereoscopic camera system. Section III presents the results of the implemented system when assessed in unknown indoor test environments. Section IV concludes this paper.

\section{METHODS}

\section{A. Stereo Disparity}

The purpose of stereo vision is to perform range measurements based on the left and right images obtained from stereoscopic cameras. Basically, an algorithm is implemented to establish the correspondence between image features in different views of the scene and then calculate the relative displacement between feature coordinates in each image. In order to produce a disparity image, the Sum of Absolute Differences (SAD) correlation algorithm in Eq.1 is used to compare a neighborhood in one stereo image to a number of neighborhoods in the other stereo image [7]

$$
S A D=\min _{d=d_{\min }}^{d_{\max }} \sum_{i=-M}^{+M} \sum_{j=-M}^{+M}\left|I_{R}(x+i, y+j)-I_{L}(x+i+d, y+j)\right| \text {, }
$$

where a window of size $(2 M+1) \times(2 M+1)$ (called a correlation mask) is centered at the coordinates of the 
matching pixels $(i, j),(x, y)$ in one stereo image, $I_{L}$ and $I_{R}$ are the intensity functions of the left and right images, and $d_{\min }, d_{\max }$ are the minimum and maximum disparities. The disparity $d_{\min }$ of zero pixels often corresponds to an infinitely far away object and the disparity $d_{\max }$ denotes the closest position of an object. If the disparity range is reduced, the system will run faster and will reduce the chance of a mismatching [8].

\section{B. Bumblebee XB3 Camera Calibrations}

The purpose of the stereoscopic camera system is to map out the visible area ahead of the wheelchair for obstacle detection, collision avoidance, and general wheelchair guidance assistance. For these reasons the wide $24 \mathrm{~cm}$ baseline was selected which utilises the outer 2 cameras of the XB3 for stereoscopic vision. This allows for wider ranges in mapping, increasing the vision range from about $42^{\circ}$ in the $12 \mathrm{~cm}$ baselines of the Bumblebee 2 and alternative $12 \mathrm{~cm}$ baseline of the Bumblebee XB3 to around $66^{\circ}$ in the $24 \mathrm{~cm}$ baseline of the Bumblebee XB3. This increased range of vision is very useful taking into consideration the constraints associated with the limited vision of these camera systems. This does however increase the dead-band distance from the camera for which no depth can be perceived, but it is still adequate to detect obstacles up to collision because the front of the wheelchair extends $50 \mathrm{~cm}$ out from the camera, which is the distance the deadband was able to be calibrated and reduced to whilst using the wide baseline.

\section{3D Point Map Generation}

Once a disparity image is produced from the processed left and right camera images, a 3D point map can be created which maps each of the depth-determined pixels from the disparity image onto a 3D plane. This can be viewed using the PGRView software which allows the 3D plane to be rotated and observed from different viewpoints. This is a very useful feature in determining where there was noise and which calibration settings improved the 3D point cloud for the purposes of accurate obstacle detection and depth analysis.

\section{2D Depth Mapping}

The 3D point maps created in the Visual C++ DLL are sorted into 3 arrays representing each pixel's (x,y,z) coordinates. Only the $\mathrm{x}$ and $\mathrm{z}$ coordinates are required for 2D depth mapping as depth maps are like 2 dimensional bird's eye view models, plotting distances against the horizontal baseline that the Bumblebee XB3 camera lies on. So the values imported in real-time from the Visual $\mathrm{C}++$ DLL are $(\mathrm{x}, \mathrm{z})$ coordinates in the form of two arrays, an $\mathrm{X}$ array and a corresponding Z-array. This corresponds such that for object depth pixel coordinates $(\mathrm{x} 0, \mathrm{z} 0),(\mathrm{x} 1, \mathrm{z} 1)$, $(x 2, z 2), \ldots,(x n, z n)$, the $X$-array $=[x 0, x 1, x 2, \ldots, x n]$ and the $\mathrm{Z}$-array $=[\mathrm{z} 0, \mathrm{z} 1, \mathrm{z} 2, \ldots, \mathrm{zn}]$. The axes are displayed on the visual depth mapping program and have $\mathrm{cm}$ units. Space is provided for memory mapping to remember objects around and behind the wheelchair, so the entire created rectanglular depth map spans $440 \mathrm{~cm}$ wide and $700 \mathrm{~cm}$ long.

\section{E. Odometry Changes and 2D Memory Depth Mapping}

Memory depth mapping is necessary for this application as the wheelchair can potentially collide with obstacles once they move out of the range of vision. So if the position of objects out of the range of vision can be recalculated and assumed to be static, then the wheelchair can use memory depth mapping to avoid collisions with these objects.

In many mobile robotic applications, position estimation methods used are usually either absolute or relative positioning [9]. The absolute positioning method generally relates the vehicles position to external environmental features, such as beacons or landmarks. However, this may involve prior knowledge or other information processes that are not required for this wheelchair as it navigates through unknown territory. The relative positioning method is more applicable here, where odometry and changes thereof can be obtained for the wheelchair as it moves. This data is acquired from wheel encoders, which individually measure rotation of the two drive wheels. This allows odometry changes in real-time to be determined, making it a simple, inexpensive, and appropriate solution for this project.

The wheelchair model assumes that wheel revolutions can be translated into linear displacement relative to the floor. This means that movements of the wheels are recorded and do not take into account conditions of slippage. However, for the short distance ranges involved, these small and rarely-occurring inaccuracies in data processing do not impact heavily on operations for this project.

\section{F. Free-space Detection and Collision Avoidance}

Standard collision avoidance is adopted for detected obstacles, however to allow the wheelchair to move through relatively narrow gaps between obstacles, the free space and the centre point of the free space is calculated to form a target for a temporary path planning routine and path following. This was made a simple detection method where the closest gap to the centre of the wheelchair's line of vision that it can fit through is chosen and the coordinate points of the objects on either side of the gap are determined.

Once a free space midpoint is detected and made a target the wheelchair needs to be able to maneuver itself in that direction. As the idea is to generally keep as much distance from obstacles as possible when moving through gaps, a potential field method was selected for collision avoidance [10]. The basis of this method is to make the target goal an attractive potential, make obstacles repulsive potentials, and combine the potential fields to allow movement towards a target whilst steering clear of obstacles. The other advantage of this method is the repulsive forces of the object points on either side of the target provide the wheelchair with a smooth approach, straightening up towards the target, preventing it from moving through a narrow gap at an awkward angle. 


\section{RESULTS}

\section{A. Hardware and Software System}

The developed system was implemented using the Bumblebee XB3 stereoscopic camera system to acquire images from the outer two cameras, providing data about the environment in front of the wheelchair. A Mac Mini was used for all computational processes; an LCD colour monitor for displaying the Graphical User Interface (GUI); wheel shaft encoders provided data about the movements of the wheelchair's two drive wheels; developed Visual C++ DLL and LabVIEW 8.5 program were used for all the image processing, wheel encoder data processing, mapping, automated decision-making for potential movements based on user selections, obstacle detections from depth maps, and wheelchair guidance control producing speed and steering output signals. These signals were sent to the NI USB-6008 for digital-to-analog conversion, and the resulting analog voltage outputs were then sent to the wheelchair motor control unit to move the wheelchair.

\section{B. 3D Point Map Generation}

When this method was tested with the generation of a 3D Point Map, the accuracy of point placement in the 3D plane was verified through the rotation of the 3D Point Map and comparison against photos taken from the corresponding angle in the actual scene. Results showed accurate object depth and position placement with the alignment of objects from the rotated 3D Point Map matching the photos actually taken from those views.

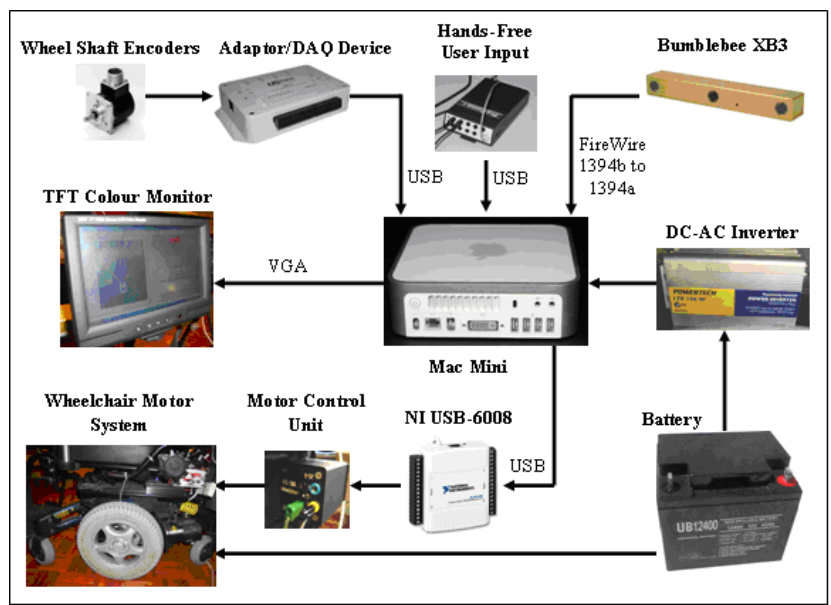

Fig. 1: Hardware System

\begin{tabular}{|c|c|c|c|}
\cline { 2 - 4 } \multicolumn{1}{c|}{} & \multicolumn{3}{c|}{ Calculated Distances } \\
\hline Actual Distance (cm) & Board & Chair & Wall \\
\hline 60 & 60 & 61 & 60 \\
\hline 80 & 81 & 83 & 79 \\
\hline 100 & 101 & 98 & 102 \\
\hline 140 & 142 & 141 & 141 \\
\hline 180 & 180 & 183 & 181 \\
\hline 220 & 218 & 218 & 220 \\
\hline 250 & 250 & 252 & 254 \\
\hline 300 & 296 & 296 & 298 \\
\hline 350 & 349 & 353 & 353 \\
\hline 400 & 396 & 395 & 398 \\
\hline 450 & 448 & 451 & 455 \\
\hline
\end{tabular}

Table 1: Actual Distance Measurements vs Calculated Distance Results

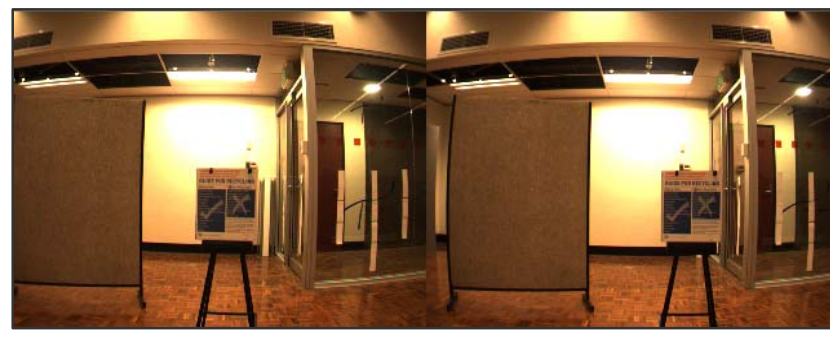

Fig. 2: Left and Right Raw Camera Images of Test Scene

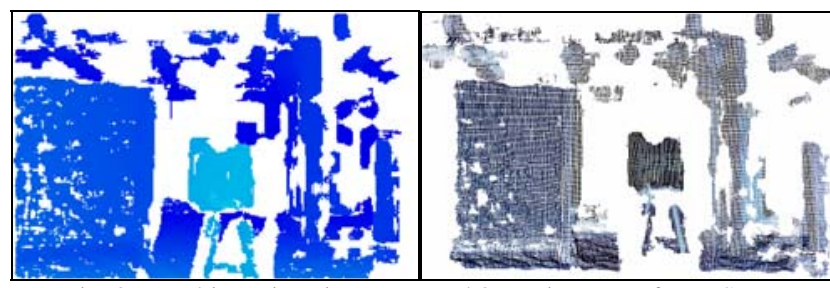

Fig. 3: Resulting Disparity Image and 3D Point Map of Test Scene

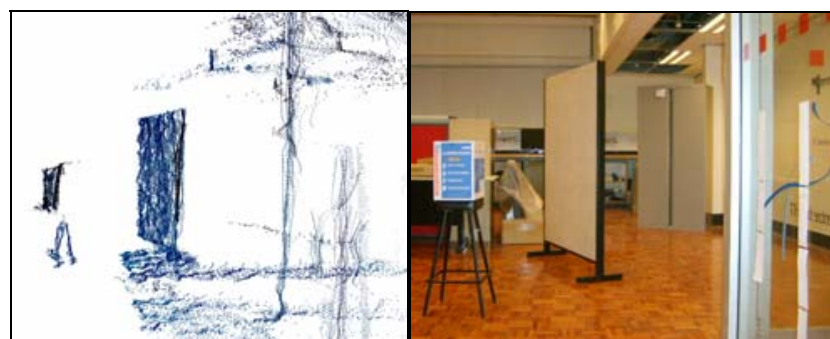

Fig. 4: Rotated 3D Point Map Comparison with Photo from the Scene

Due to the optimisation of the 3D point clouds based on camera calibrations, the first set of tests on the depth mapping program provided accurate results that did not require further optimisation. These tests involved a board and a chair being placed at set distances as well as the wheelchair and Bumblebee XB3 camera being moved toward a wall at set distances. The actual distances measured with a measuring tape versus the calculated distances from the Z-array data are displayed in Table 1.

\section{Memory 2D Depth Mapping}

Observation of the memory depth maps, during wheelchair maneuvering, showed accurate repositioning of obstacle depth data once they had moved outside the vision range, indicated on the 2D Depth Map with straight lines.

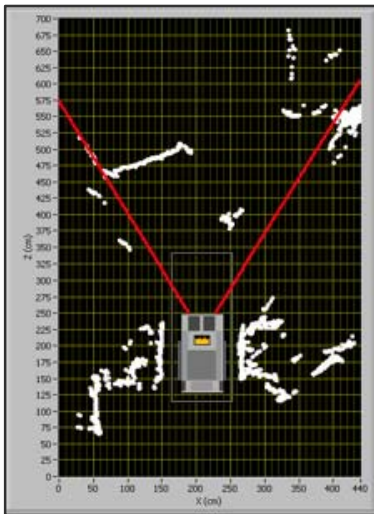

Fig. 5: Memory Mapping Observation / Measurement Test Results 


\begin{tabular}{|c|c|c|}
\hline Measured Feature & Actual Measurement & Memory Map Calculation \\
\hline Length of desk on the left & 84 & 89 \\
\hline Length of desk on the right & 84 & 81 \\
\hline Width between desks & 121 & 119 \\
\hline Gap between left desk and WC & 29 & 28 \\
\hline Gap between right desk and WC & 27 & 26 \\
\hline $\begin{array}{c}\text { Length from front of left desk } \\
\text { to front of WC }\end{array}$ & 18 & 16 \\
\hline $\begin{array}{c}\text { Length from front of right desk } \\
\text { to front of WC }\end{array}$ & 18 & 20 \\
\hline $\begin{array}{c}\text { Length from back of WC } \\
\text { to back of left desk }\end{array}$ & 23 & 20 \\
\hline $\begin{array}{c}\text { Length from back of WC } \\
\text { to back of right desk }\end{array}$ & 23 & 24 \\
\hline
\end{tabular}

Table 2: Memory Depth Map Calculations vs Actual Feature Measurements

The idea of this test was simply to observe the accuracy of recalculated and repositioned points on the map once outside the range of vision of the Bumblebee XB3 camera system. It can be seen from the screen shots of the 2D depth map that an icon of the wheelchair, along with its vision limits drawn in red in Fig. 5, has been placed on the 2D depth map. The dimensions and positioning of these on the map were accurate as the 2D depth map dimensions and axes were designed and calibrated around this icon. As the wheelchair moved forward in the scene it recalculated the positions of all the static objects it had mapped when in the lines of vision, and accurately repositioned all of these accordingly on the map as it moved. Once the wheelchair was moved forward into the position shown in Fig. 6, measurements were taken to test the repositioned objects in the scene which had moved outside the range of vision.

It can be seen here that all calculations in the memory depth map are very close to the actual measurements, all within an accuracy of $\pm 5 \mathrm{~cm}$. The next test was on the automated obstacle detection and wheelchair guidance and collision-avoidance system.

From the test scene in Fig. 8 the wheelchair must first detect that the first gap to the left is large enough (greater than $80 \mathrm{~cm}$ ), calculate the midpoint of the gap, maneuver itself toward and through that gap, then detect the second gap ahead and to the right, maneuver itself past the first gap and toward the second gap, then proceed through into the larger area where it then has to avoid a final object positioned ahead to the right. With the gaps being reduced to $0.9 \mathrm{~m}$ wide, the wheelchair did not have much room for error, however the guidance system allowed it to autonomously navigate its way through such a course smoothly and without collision.

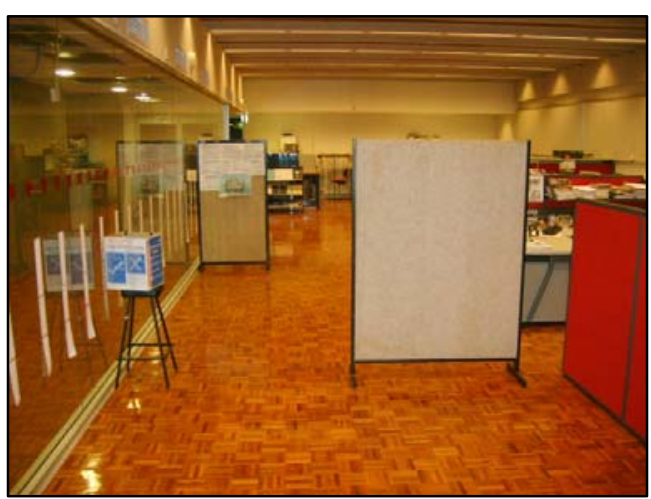

Fig. 6: Wheelchair Guidance System: Obstacle Course Test

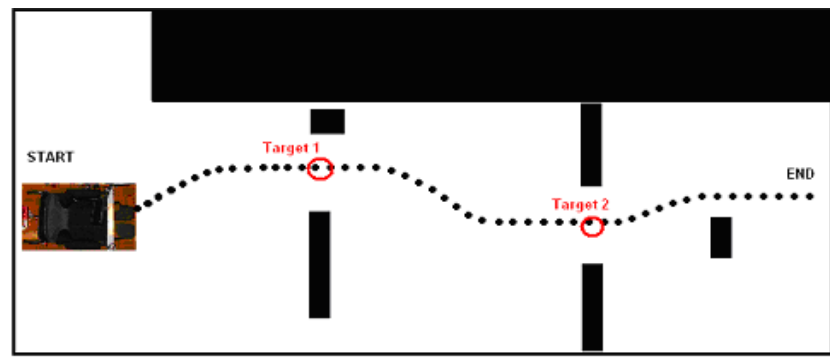

Fig. 7: Navigation Path taken by the Wheelchair in Obstacle Course Test

\section{CONCLUSION}

This system has shown that stereoscopic cameras, such as the Point Grey Research Bumblebee XB3 system, can be used to allow a semi-autonomous wheelchair to assist the user with effective guidance assistance when navigating in unknown indoor environments. Due to the narrow vision of this current system it is only useful in static environments as it remembers placement of obstacles outside its vision range. This will, however, be improved from here to allow efficient power wheelchair guidance assistance for hands-free control technology in indoor and outdoor, static and dynamic environments.

\section{REFERENCES}

[1] R. C. Simpson, "Smart Wheelchairs: A Literature Review", Journal of Rehabilitation Research \& Development, vol. 42, pp. 423-436, 2005.

[2] Y. Adachi, K. Goto, Y. Matsumoto, and T. Ogasawara, "Development of Control Assistant System for Robotic Wheelchair-estimation of User's Behavior Based on Measurements of Gaze and Environment", Proceedings of IEEE International Symposium on Computational Intelligence in Robotics and Automation, pp. 538 - 543, 2003.

[3] S. P. Levine, D. A. Bell, A. J. Lincoln, R . C. Simpson, Y. Koren, J. Borenstein, "The NavChair Assistive Wheelchair Navigation System", IEEE Transactions on Rehabilitation Engineering, vol. 7, no. 4, 1999.

[4] R. C. Simpson, D. Poirot, F. Baxter, "The Hephaestus Smart Wheelchair System", IEEE Transactions on Neural Systems and Rehabilitation Engineering, vol. 10, no. 2, 2002.

[5] U. Frese, P. Larsson, T. Duckett, "A Multigrid Algorithm for Simultaneous Localization and Mapping", IEEE Transactions on Robotics, vol. 21, no.2, pp. 1-12, 2005.

[6] A. J. Davidson, I. D. Reid, N. D. Molton, O. Stasse, "MonoSLAM: Real-Time Single Camera SLAM", IEEE Transactions on Pattern Analysis and Machine Intelligence, vol. 29, 2007.

[7] C. Watman, D. Austin, N. Barnest, G. Overett, and S. Thompson, "Fast Sum of Absolute Differences Visual Landmark Detector", Proceedings of the IEEE International Conference on Robotics 8 Automation, 2004, pp. 4827-4832.

[8] T. H. Nguyen, J. S. Nguyen, D. M. Pham, and H. T. Nguyen, "RealTime Obstacle Detection for an Autonomous Wheelchair Using Stereoscopic Cameras," in The 29th Annual International Conference of the IEEE Engineering in Medicine and Biology Society, 2007, pp. $4775-4778$.

[9] C. M. Wang, "Location Estimation and Uncertainty Analysis for Mobile Robots", IEEE International Conference on Robotics and Automation, Michigan, pp. 1231-1235, 1988.

[10]H. Igarashi and M. Kakikura, "Path and Posture Planning for Walking Robots by Artificial Potential Field Method", Proceedings of the 2004 IEEE International Conference on Robotics and Automation, pp. 21652170, 2004.

[11] G. N. Desouza and A. C. Kak, "Vision for Mobile Robot Navigation: A Survey", IEEE Transactions on Pattern Analysis and Machine Intelligence, vol. 24, pp. 237-267, 2002.

[12]D. Murray and J. J. Little, "Using Real-Time Stereo Vision for Mobile Robot Navigation", Autonomous Robots, vol. 8, pp. 161-171, 2000. 